\title{
Percepción de Egresados y Profesorado sobre la Implicación del Alumnado en la Evaluación y la Calificación en Educación Superior
}

\section{Perception of Graduates and Teachers on Participation in Assessment in Higher Education}

\author{
Laura Cañadas * \\ María Luisa Santos-Pastor \\ Francisco Javier Castejón
}

Universidad Autónoma de Madrid, España

\begin{abstract}
La evaluación es uno de los elementos metodológicos más importantes en la enseñanza universitaria. Tras las reformas del Espacio Europeo de Educación Superior, la evaluación debe adquirir un carácter formativo para dar respuesta a las nuevas demandas. Por ello, para conocer si estos cambios están teniendo lugar, esta investigación busca: (a) conocer la percepción de egresados y profesorado respecto a los instrumentos de evaluación utilizados y la implicación del alumnado en su calificación; (b) valorar si existen diferencias entre la percepción de egresados y profesorado en estos temas; y (c) conocer si aquellos que perciben una mayor participación en los procesos de calificación también perciben una utilización de instrumentos de evaluación más variada. Participaron 491 egresados y 344 docentes de 20 Facultades de Educación y de Ciencias de la Actividad Física y el Deporte. Los resultados muestran que, los egresados consideran que se utilizan en mayor medida una variedad de instrumentos de evaluación que el profesorado, siendo únicamente en los exámenes orales, los exámenes dejando disponer de documentos y los ensayos donde no aparecen diferencias estadísticamente significativas entre ambos grupos; y (b) los egresados presentan una relación positiva entre la utilización de las formas de calificación participativas y la utilización de la mayoría de los instrumentos de evaluación, mientras que el profesorado relaciona estas formas de calificación inversamente con la utilización de exámenes.
\end{abstract}

Palabras clave: Educación superior; Evaluación formativa; Instrumentos evaluación; Calificación; Formación inicial del profesorado.

\begin{abstract}
Assessment is one of the most important methodological elements in university teaching. After European Higher Education reform, assessment should acquire a formative perspective so it can respond to the new demands. So, in order to know if those changes are taking place, the aims of this research are (a) examine graduates' and teachers' perception on the use of assessment tools and students' grading participation; (b) analyze if there are differences between graduates and teachers perception on those topics and; (c) examine if those who perceived more participation in grading process also perceived a more varied use of assessment instruments. 491 graduates and 344 teachers from 20 faculties of Education and Physical Activity and Sport Sciences participated in the study. Results of this research show that graduates consider that a variety of assessment instruments are more widely used than teachers, only there are not statistical differences in oral exams, exams leaving documents and essays between both groups; and (b) graduates present a positive relationship between the use of participatory grading forms and the use of most assessment instruments, while teachers relate these forms of grading inversely with the use of exams.
\end{abstract}

Keywords: Higher education; Formative assessment; Assessment instruments; Grading; Pre-service teacher education.

*Contacto: laura.cannadas@uam.es

issn: 1989-0397

www.rinace.net/riee/

https://revistas.uam.es/riee
Recibido: $\quad 16$ de enero de 2019

$1^{\text {a }}$ Evaluación: 18 de febrero de 2019

$2^{\text {a }}$ Evaluación: 25 de marzo de 2019

Aceptado: $\quad 01$ de abril de 2019 


\section{Introducción}

Uno de los cambios sufridos por la universidad, en lo que a materia educativa se refiere, ha sido la convergencia hacia el Espacio Europeo de Educación Superior (EEES) para homogeneizar el sistema universitario (González, 2006). Entre las actuaciones emprendidas dentro del EEES destaca: a) el establecimiento de titulaciones compatibles y comparables; b) la adopción de una estructura de los estudios basada en dos ciclos principales (Grado y Posgrado); c) la definición de sistemas de evaluación de la calidad de acuerdo a unos estándares internacionales; d) el establecimiento de un sistema de créditos transferibles; e) la promoción de la cooperación europea en calidad y desarrollo de criterios y metodologías comparables; y f) la promoción de la movilidad de los estudiantes, investigadores y profesores (Campbell y Van der Wende, 2000).

Entre las principales líneas de actuación dentro del proceso de convergencia, destaca la creación del "sistema europeo de transferencia y acumulación de créditos (ECTS)" (European Communities, 2009). Este sistema tiene como objetivo facilitar el diseño, planificación y desarrollo de las titulaciones, así como el reconocimiento y convalidación entre las diferentes unidades de aprendizaje como asignaturas, módulos o materias (Comisión Europea, 2004, 2011). El ECTS es un sistema basado en el estudiante, centrado en su carga de trabajo para la consecución de los objetivos de un programa (Espinosa, Jiménez, Olabe y Basogain, 2006). Dichos objetivos se especifican en términos de resultados de aprendizaje y de las competencias a adquirir (Comisión Europea, 2004). Por tanto, con esta nueva perspectiva, no cambia solo la estructura de las enseñanzas universitarias, sino que también se produce un cambio de mentalidad en el planteamiento del proceso de enseñanza y aprendizaje centrando el objetivo en el estudiante (RD 1393/2007). También las metodologías docentes deben dejar de lado los planteamientos clásicos centrados en la transmisión del conocimiento por parte del profesorado, para dar paso a metodologías activas en las que el alumnado, guiado por los docentes, sea responsable de sus aprendizajes, centrándose en aprender a aprender (Palomares, 2007).

Uno de los aspectos sobre los que será necesario intervenir para responder a las demandas del EEES es el tipo de evaluación del alumnado (Delgado, 2005; González, 2006). La evaluación es uno de los elementos claves del proceso formativo que se vincula directamente con la calidad de la enseñanza, además de ser una herramienta esencial para conseguir un aprendizaje efectivo (Biggs y Tang, 2007; Bonsón y Benito, 2007; Brown y Glasner, 2007).

\section{Fundamentación teórica}

Tradicionalmente, la evaluación se ha entendido como la valoración del nivel de consecución de los objetivos previstos al finalizar un periodo de enseñanza y en qué grado se han conseguido estos. La evaluación tradicional se orienta a la rendición de cuentas, dirigida por la calificación como certificación de resultados. La calificación es un acto administrativo donde se trata de "convalidar" entre lo que se aprende y lo que se constata (Álvarez, 2003); es decir, traduce aprendizajes en marcas, notas, calificaciones. Esta queda establecida con una prueba final, por lo general un examen, para demostrar lo que sabe o no un estudiante de una asignatura concreta (Calderón y Escalera, 2008; Trillo y Porto, 
1999). Actualmente, desde el marco del EEES, esta forma de evaluar y calificar no tiene cabida. Se demanda una evaluación para el aprendizaje (Brown, 2004; Stiggins, 2005).

A nuestro entender, y siguiendo las actuales propuestas, dicha evaluación debe tener un carácter formativo (Brown y Glasner, 2007; Sambell, McDowell y Montgomery, 2013), que permita utilizar esta para que el alumnado conozca y pueda planificar sus actividades de aprendizaje ayudándole a mejorar en los mismos (López-Pastor, 2006). Para que la evaluación formativa consiga cumplir su función, debe ser un proceso sistemático de recogida de información sobre el aprendizaje de los estudiantes mientras el proceso de instrucción está teniendo lugar (Black y Wiliam, 1998, 2009; Heritage, 2007), y debe utilizarse para proporcionar una retroalimentación al alumnado sobre cómo lo está haciendo y qué puede hacer para mejorar o aprender más (Crooks, 1988; Sadler, 1989; Wiliam, 2006; Yorke, 2003). En los procesos de evaluación formativa se utilizan distintos tipos de instrumentos de evaluación para conseguir una mayor y más variada información sobre el aprendizaje del alumnado. Otro de los aspectos característicos de la evaluación formativa es la participación del alumnado en los procesos de evaluación y calificación. Se fomenta la utilización de la auto-evaluación (el alumnado se evalúa a sí mismo), la evaluación entre pares o co-evaluación (el alumnado evalúa a sus compañeros y compañeras) y la evaluación compartida entre el profesorado y el alumnado (Dochy, Segers y Sluijsmans, 1999; Gessa, 2011; Rodríguez, Ibarra y García, 2013), junto a los respectivos procesos de calificación que cada una de estas formas de evaluación pueden llevar asociados: auto-calificación, calificación entre compañeros o a través de una calificación dialogada entre ambos agentes (Ibarra y Rodríguez, 2014; López-Pastor, 2006, 2017).

Respecto a los aspectos que ayudan a valorar si se está produciendo este cambio en la forma de plantear la evaluación, se encuentran tanto el tipo como la variedad de instrumentos de evaluación utilizados, así como las estrategias de participación del alumnado en los procesos de evaluación y calificación. Los instrumentos de evaluación son "las herramientas de las que se sirve el profesorado para obtener la información que utiliza en la evaluación” (Castejón, Capllonch, González y López-Pastor, 2009, p. 65). Según Padilla y Gil (2008), en este nuevo contexto en el que nos encontramos ya no será suficiente con la utilización del examen (o del examen y el trabajo escrito) para evaluar al alumnado, sino que será necesario el uso de una mayor diversidad de instrumentos. Por otra parte, la calificación entendida como una nota alfanumérica que se asigna a cada persona (Hamodi, López-Pastor y López, 2015), siempre ha sido considerada como una tarea única del docente (hetero-calificación), pero bajo este nuevo marco es necesaria la implicación del alumnado, ya sea interviniendo en su propia calificación o en la de otros estudiantes (Ibarra, Rodríguez y Gómez, 2012). Hacerles partícipes en el proceso de calificación fomentará que el alumnado sea consciente de sus aprendizajes, así como que tome responsabilidad dentro del proceso de enseñanza y aprendizaje.

Es importante conocer si durante el periodo transcurrido desde la implantación de las políticas del EEES se ha percibido un cambio en la utilización de una mayor diversidad de instrumentos de evaluación, así como una mayor participación del alumnado en los procesos de calificación. Para ello, no es suficiente con conocer qué es lo que el profesorado universitario dice hacer, sino también qué es lo que piensan quienes han vivido su formación inicial durante el periodo de implantación de los nuevos Grados, es decir, los egresados. Los egresados son un grupo formado por personas que ya han terminado sus 
estudios universitarios y que pueden aportar información sobre las enseñanzas recibidas durante su formación recordándola con bastante claridad. Además, la visión que estos tienen sobre las enseñanzas recibidas puede tener relación directa con la visión que tiene el alumnado (Arribas, Manrique y Tabernero, 2016; Gutiérrez, Pérez y Pérez, 2013).

Las investigaciones que han valorado y comparado la percepción de profesorado y egresados respecto a la utilización de instrumentos de evaluación y la forma de calificación, coinciden en la poca variedad de instrumentos de evaluación utilizados, así como en la discrepancia en la mayoría de los casos entre estos dos grupos respecto a los instrumentos de evaluación más y menos empleados (Arribas et al., 2016; Cañadas, Santos-Pastor y Castejón, 2018a; Gutiérrez et al., 2013; Hamodi et al., 2015). Estos estudios concuerdan en que la participación durante la formación inicial en procesos de auto-calificación, calificación entre compañeros o calificación dialogada es muy baja predominando para ambos grupos los procesos de hetero-calificación. Otros estudios se han centrado únicamente en conocer la percepción de un grupo poblacional (docentes universitarios, egresados, alumnado) sobre la utilización de los diferentes instrumentos de evaluación y las formas de calificación empleadas. Por ejemplo, el estudio de Cañadas, Santos-Pastor y Castejón (2018b) valora la percepción que tiene una muestra de más de 2000 estudiantes universitarios sobre los instrumentos de evaluación empleados y las formas de calificación utilizadas, encontrando que, los exámenes tipo test y los trabajos escritos son los instrumentos más utilizados y predomina la hetero-calificación. Mientras que el estudio de Martínez, Castejón y Santos-Pastor (2012) encuentra que existe una predominancia en la utilización de exámenes de preguntas abiertas.

Sin embargo, ninguno de los estudios revisados ha analizado si existe una relación entre la forma de calificación utilizada y el empleo de instrumentos de evaluación, valorando si las formas de calificación participativas se asocian con un mayor número de instrumentos que la forma de calificación tradicional, basada, principalmente, en la utilización única y exclusiva de un examen final.

Por ello, los objetivos de esta investigación son: a) conocer si existen diferencias entre la percepción de egresados y profesorado respecto a los instrumentos de evaluación empleados y la implicación del alumnado en su calificación; y b) conocer si aquellos que valoran una mayor implicación en los procesos de calificación también perciben una utilización de instrumentos de evaluación más variada.

\section{Método}

\subsection{Participantes}

Para la selección de los participantes de esta investigación se utilizó un muestreo incidental no probabilístico. Se recogieron datos de: (a) 491 egresados (40,7 \% mujeres) diplomados en Educación Física, Graduados en Primaria con especialidad en Educación Física, Licenciados y Graduados en Ciencias de la Actividad Física y el Deporte y; (b) 344 docentes (43,3 \% mujeres) que imparten su docencia en asignaturas de Educación Física de los Grados de Magisterio en Educación Primaria y Magisterio en Educación Infantil, y en el Grado en Ciencias de la Actividad Física y el Deporte. Los datos de egresados y profesorado para la presente investigación fueron recogidos en 20 centros de Educación Superior, entre los que se encuentran Facultades de Educación y de Profesorado y 
Facultades de Ciencias de la Actividad Física y el Deporte (Figura 1). Los datos se recogieron entre los años 2014 y 2015 a través de un cuestionario on-line.

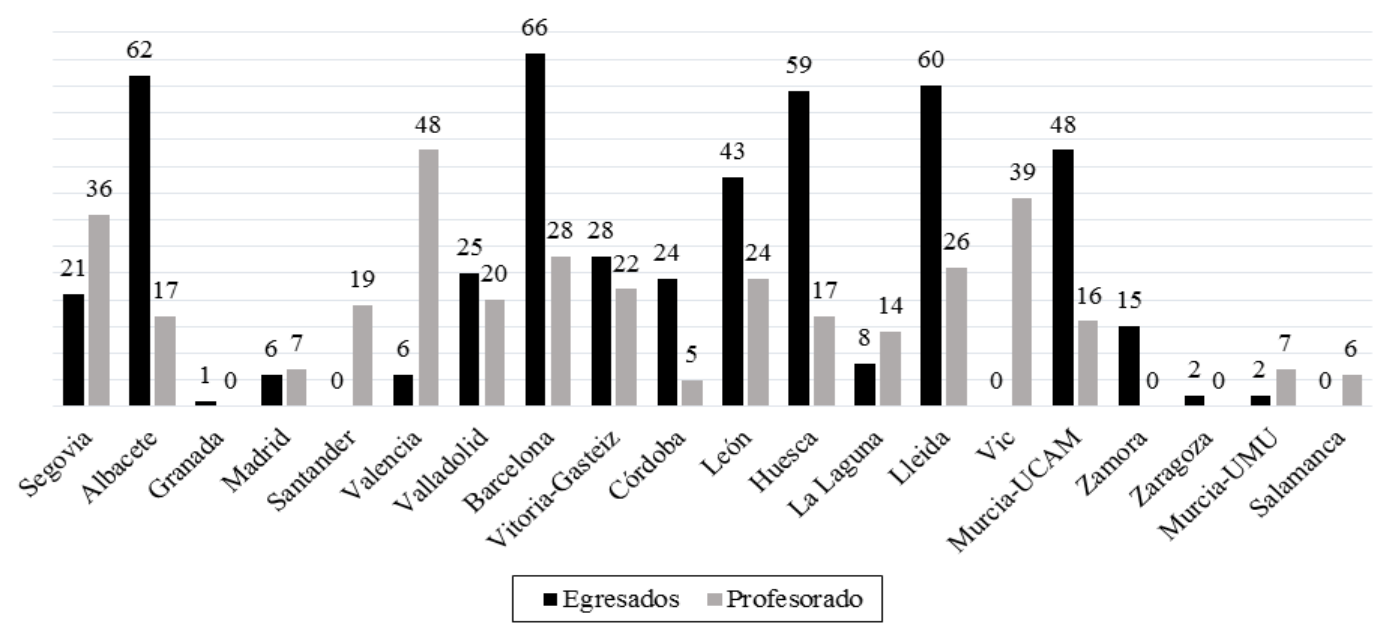

Figura 1. Número de participantes en el estudio en función de su procedencia Fuente: Elaboración propia.

\subsection{Instrumentos}

Para la recogida de información se utilizó un cuestionario validado sobre competencias docentes y evaluación formativa que se desarrolló para el proyecto "Las competencias docentes en la formación inicial del profesorado de Educación Física” del cual forma parte esta investigación (Palacios, López-Pastor y Fraile-Aranda, 2018) y que ha sido desarrollado por la Red de Evaluación Formativa y Compartida en Educación Superior (López-Pastor, Castejón, Sicilia, Navarro y Webb, 2011; López-Pastor, Martínez y Julián, 2007; https://redevaluacionformativa.wordpress.com/). A las dos poblaciones participantes en el estudio se les pasó un cuestionario diferenciado. Ambos cuestionarios (para egresados y profesorado) incluían las mismas preguntas, pero aludiendo al grupo poblacional que se iba a estudiar. Para esta investigación se emplearon dos preguntas. Una referida a los instrumentos de evaluación utilizados durante el desarrollo de las asignaturas de la carrera y otra a la forma de calificar del profesorado universitario en sus asignaturas.

- Pregunta sobre la frecuencia de utilización de instrumentos de evaluación en las asignaturas. Esta está formada por ítems que recogen instrumentos de evaluación que van desde pruebas más tradicionales como diferentes tipologías de exámenes (tipo test, de preguntas abiertas, de preguntas cerradas, de preguntas cortas, orales, dejando disponer de documentos) pasando por trabajos escritos, portafolios, pruebas físicas o proyectos tutelados.

- Pregunta referida a cómo se han establecido las calificaciones en las diferentes asignaturas. Esta recoge cuatro posibilidades: a) calificación decidida únicamente por el profesor/a o hetero-calificación; b) el alumnado se auto-calificaba; c) la nota se ponía de forma dialogada entre el profesorado y el alumnado; y d) el alumnado calificaba entre compañeros. 
Cada uno de los ítems se valoraba con una escala Likert que iba desde el o (Nada) hasta 4 (Mucho).

\subsection{Procedimientos}

El estudio sigue las directrices éticas de la American Psychological Association (2010) con respecto a la confidencialidad y anonimato de los participantes, así como al consentimiento informado de los mismos. Se hizo llegar el cuestionario vía correo electrónico a los posibles participantes de las diferentes universidades participantes, informándoles de la finalidad de la investigación, solicitando su participación, y asegurando la confidencialidad de las respuestas obtenidas.

\subsection{Análisis de datos}

Los análisis se realizaron con el paquete estadístico SPSS v. 21 para Windows. La frecuencia de uso de diferentes instrumentos de evaluación y diferentes formas de calificación se presenta como Media (Desviación típica) y porcentajes. La diferencia de pensamiento en la utilización de instrumentos de evaluación y en las formas de calificación entre el profesorado y los egresados se valoró con una t de student. Con el objetivo de conocer la relación entre procesos de calificación y uso de instrumentos de evaluación, se tomaron las variables de forma continua y se analizaron los datos a través de una correlación de Pearson. Estos datos se analizaron de forma independiente para egresados y profesorado. Se presenta el valor de la correlación $(\mathrm{r})$ y de la p. El nivel de significatividad se estableció en $\mathrm{p}<0,05$ para todos los análisis.

\section{Resultados}

El Cuadro 1 muestra la opinión de egresados y profesorado sobre la frecuencia de uso de una variedad de instrumentos de evaluación y sobre las formas de calificación utilizadas durante la formación inicial del profesorado, así como la diferencia de percepción entre ambos grupos en cada una de estas. En cuanto a los instrumentos de evaluación, existen diferencias entre el pensamiento de profesorado y egresados en todas las variables menos en "exámenes de preguntas abiertas", y "ensayos a partir de textos o materiales audiovisuales", con una frecuencia de utilización media (entre 2,13 y 2,57), y "exámenes dejando disponer de documentos", y "exámenes orales" con una frecuencia media de utilización baja $(<1,15)$. En la calificación, aunque ambos grupos coinciden en que la forma de calificación más utilizada es la hetero-calificación, y que las formas de calificación participativas son poco usadas, los egresados reportan valores medios más altos en la hetero-calificación y más bajos en las formas de calificación participativas, en comparación con el profesorado (todos, $\mathrm{p}<0,001$ ).

Los instrumentos que los egresados perciben que más han sido utilizados durante su formación inicial son los trabajos escritos $(48,8 \%)$ y los exámenes tipo test (40,6\%). Los instrumentos que se perciben que no se usan nunca son los exámenes orales $(43,2 \%)$ y los exámenes escritos dejando disponer de documentos (38,7\%). La hetero-calificación se presenta como la forma de calificación más utilizada ( $>80 \%$ responden bastante o mucho), mientras que las formas de calificación que implican la participación del alumnado obtienen más de un $60 \%$ al sumar los grupos de poco y nada utilizada. 
$\underline{\text { Cuadro 1. Medias y porcentajes de utilización de instrumentos de evaluación y tipos de calificación de egresados y profesorado }}$

\begin{tabular}{|c|c|c|c|c|c|c|c|c|c|c|c|c|c|}
\hline & \multicolumn{5}{|c|}{ EGRESADOS } & \multicolumn{8}{|c|}{ PROFESORADO } \\
\hline & $\mathrm{M}(\mathrm{DT})$ & $\mathrm{O}$ & 1 & 2 & 3 & 4 & $\mathrm{M}(\mathrm{DT})$ & $\mathrm{O}$ & 1 & 2 & 3 & 4 & $p$ \\
\hline$n$, & 488 & & & & & & 344 & & & & & & \\
\hline \multicolumn{14}{|l|}{ Instrumentos de evaluación } \\
\hline Examen tipo test & $2,95(1,10)$ & 2,3 & 10,2 & 18,4 & 28,5 & 40,6 & $1,58(1,57)$ & 42,2 & 8,7 & 14,8 & 17,2 & 17,2 & $<0,001$ \\
\hline Examen de preguntas abiertas & $2,57(1,08)$ & 4,1 & 12,9 & 26,2 & 35,9 & 20,9 & $2,41(1,37)$ & 16,6 & 7,8 & 17,7 & 33,4 & 24,4 & 0,086 \\
\hline Examen de preguntas cortas & $2,63(0,98)$ & 2,7 & 10,0 & 27,5 & 41,2 & 18,6 & $2,27(1,31)$ & 17,2 & 9,3 & 18,3 & 40,1 & 15,1 & $<0,001$ \\
\hline Examen de preguntas cerradas & $2,01(1,16)$ & 9,8 & 25,8 & 28,7 & 24,6 & 11,1 & $0,97(1,19)$ & 50,6 & 19,8 & 14,5 & 11,9 & 3,2 & $<0,001$ \\
\hline Exámenes escritos dejando documentos & $0,99(1,04)$ & 38,7 & 36,5 & 15,4 & 6,4 & 3,1 & $1,13(1,21)$ & 49,1 & 13,1 & 21,2 & 9,3 & 7,3 & 0,101 \\
\hline Exámenes orales & $0,93(1,06)$ & 43,2 & 33,6 & 13,3 & 6,6 & 3,3 & $1,00(1,29)$ & 53,8 & 15,4 & 14,2 & 10,2 & 6,4 & 0,410 \\
\hline Pruebas prácticas de carácter físico & $2,20(1,22)$ & 10,2 & 19,7 & 26,0 & 28,3 & 15,8 & $0,94(1,40)$ & 63,1 & 8,4 & 7,6 & 12,8 & 8,1 & $<0,001$ \\
\hline Portafolios & $2,12(1,27)$ & 13,7 & 17,6 & 28,3 & 23,6 & 16,8 & $1,77(1,59)$ & 36,0 & 11,0 & 12,5 & 20,3 & 20,1 & 0,001 \\
\hline Cuadernos de campo & $2,55(1,06)$ & 3,1 & 14,8 & 25,4 & 37,5 & 19,3 & $1,97(1,50)$ & 26,7 & 12,2 & 19,2 & 21,2 & 20,6 & $<0,001$ \\
\hline Informes o trabajos escritos & $3,26(0,89)$ & 0,6 & 5,1 & 10,5 & 35,0 & 48,8 & $3,04(1,03)$ & 4,4 & 4,1 & 12,8 & 40,4 & 38,4 & 0,001 \\
\hline $\begin{array}{l}\text { Ensayos a partir de textos o materiales } \\
\text { audiovisuales }\end{array}$ & $2,13(1,22)$ & 8,4 & 20,9 & 31,1 & 28,5 & 11,1 & $2,22(1,39)$ & 19,2 & 10,5 & 19,5 & 31,4 & 19,5 & 0,342 \\
\hline Proyectos de aprendizaje tutorados & $1,95(1,21)$ & 12,7 & 25,6 & 27,9 & 21,9 & 11,9 & $2,29(1,40)$ & 17,7 & 11,0 & 19,2 & 28,8 & 23,3 & $<0,001$ \\
\hline \multicolumn{14}{|l|}{ Tipos de calificación utilizados } \\
\hline Hetero-calificación & $3,25(0,82)$ & 1,0 & 2,3 & 10,9 & 42,4 & 43,4 & $2,94(0,90)$ & 2,0 & 4,7 & 17,4 & 48,8 & 27,0 & $<0,001$ \\
\hline Auto-calificación & $1,30(1,10)$ & 25,4 & 39,1 & 19,1 & 12,5 & 3,9 & $1,78(1,14)$ & 16,9 & 23,3 & 28,5 & 27,3 & 4,1 & $<0,001$ \\
\hline Calificación dialogada & $1,04(1,13)$ & 39,1 & 35,0 & 12,9 & 8,2 & 4,7 & $1,54(1,22)$ & 22,1 & 32,8 & 21,5 & 15,7 & 7,8 & $<0,001$ \\
\hline Calificación entre compañeros & $1,26(1,02)$ & 24,6 & 41,0 & 20,9 & 11,3 & 2,3 & $1,54(1,23)$ & 25,6 & 27,0 & 20,9 & 20,9 & 5,5 & $<0,001$ \\
\hline
\end{tabular}

Nota: En negrita las diferencias significativas.

Fuente: Elaboración propia. 
Por parte del profesorado, los instrumentos que se consideran muy utilizados son los trabajos escritos $(38,4 \%)$ y los exámenes de preguntas abiertas $(24,4 \%)$. Si tenemos en cuenta de forma conjunta el porcentaje de bastante y mucho, a estos dos instrumentos se sumaría como otro de los más utilizados los exámenes de preguntas cortas $(55,2 \%)$. En cuanto a los instrumentos que el profesorado considera que no usa nunca aparecen las pruebas de carácter físico en primer lugar $(63,1 \%)$, seguido de los exámenes orales $(53,8 \%)$, los exámenes de preguntas cerradas $(50,6 \%)$ y los exámenes escritos dejando disponer de documentos $(49,1 \%)$. Similares resultados se obtienen si juntamos la categoría de nada y poco. En cuanto a la calificación, el profesorado considera que usa bastante la heterocalificación (48,8\%), que sumada al porcentaje de mucho, supera el $75 \%$. La calificación dialogada y la calificación entre compañeros obtienen sus mayores porcentajes en la respuesta de poco, un $32,8 \%$ y un $27 \%$, respectivamente. En el caso de la auto-calificación, el porcentaje más alto se encuentra al sumar los grupos de poco y nada (40,2\%).

El Cuadro 2 presenta los resultados de los egresados sobre la correlación entre el tipo de procesos de calificación aplicados y los instrumentos de evaluación utilizados. La heterocalificación muestra una correlación significativa y positiva con el uso de examen de preguntas abiertas $(r=0,126 ; p=0,005)$. Los procesos de auto-calificación y calificación dialogada muestran también una relación significativa y positiva en todas las variables excepto en los exámenes tipo test y los trabajos escritos (ambos, $p>0,05$ ). Lo mismo ocurre con la calificación entre compañeros, donde aparte de estos dos ítems tampoco aparece relación en los exámenes de preguntas abiertas $(p=0,200)$.

Cuadro 2. Correlación entre instrumentos de evaluación y tipo de evaluación en egresados

\begin{tabular}{|c|c|c|c|c|c|c|c|c|}
\hline & \multicolumn{2}{|c|}{$\begin{array}{c}\text { HETERO- } \\
\text { CALIFICACIÓN }\end{array}$} & \multicolumn{2}{|c|}{$\begin{array}{c}\text { AUTO- } \\
\text { CALIFICACIÓN }\end{array}$} & \multicolumn{2}{|c|}{ Cal. Dialogada } & \multicolumn{2}{|c|}{$\begin{array}{c}\text { CALIFICACIÓN } \\
\text { ENTRE COMPAÑEROS }\end{array}$} \\
\hline & $\boldsymbol{r}$ & $p$ & $\boldsymbol{r}$ & $p$ & $r$ & $p$ & $\boldsymbol{r}$ & $p$ \\
\hline Examen tipo test & 0,081 & 0,074 & $-0,066$ & 0,148 & $-0,056$ & 0,214 & 0,004 & 0,923 \\
\hline $\begin{array}{l}\text { Examen de } \\
\text { preguntas abiertas }\end{array}$ & 0,126 & 0,005 & 0,109 & 0,016 & 0,105 & 0,021 & 0,058 & 0,200 \\
\hline $\begin{array}{l}\text { Examen de } \\
\text { preguntas cortas }\end{array}$ & 0,051 & 0,259 & 0,138 & 0,002 & 0,147 & 0,001 & 0,186 & $<0,001$ \\
\hline $\begin{array}{l}\text { Examen de } \\
\text { preguntas cerradas }\end{array}$ & 0,010 & 0,829 & 0,123 & 0,007 & 0,208 & $<0,001$ & 0,155 & 0,001 \\
\hline $\begin{array}{l}\text { Exámenes escritos } \\
\text { dejando disponer de } \\
\text { documentos }\end{array}$ & $-0,037$ & 0,414 & 0,334 & $<0,001$ & 0,429 & $<0,001$ & 0,430 & $<0,001$ \\
\hline Exámenes orales & 0,044 & 0,332 & 0,296 & $<0,001$ & 0,319 & $<0,001$ & 0,335 & $<0,001$ \\
\hline $\begin{array}{l}\text { Pruebas prácticas de } \\
\text { carácter físico }\end{array}$ & 0,076 & 0,091 & 0,253 & $<0,001$ & 0,185 & $<0,001$ & 0,269 & $<0,001$ \\
\hline Portafolios & 0,028 & 0,534 & 0,209 & $<0,001$ & 0,192 & $<0,001$ & 0,235 & $<0,001$ \\
\hline $\begin{array}{l}\text { Cuadernos de } \\
\text { campo (o fichas de } \\
\text { sesiones prácticas) }\end{array}$ & $-0,003$ & 0,948 & 0,208 & $<0,001$ & 0,159 & $<0,001$ & 0,177 & $<0,001$ \\
\hline $\begin{array}{l}\text { Informes o trabajos } \\
\text { escritos }\end{array}$ & 0,048 & 0,287 & 0,083 & 0,068 & 0,026 & 0,572 & 0,068 & 0,131 \\
\hline $\begin{array}{l}\text { Ensayos a partir de } \\
\text { textos o materiales } \\
\text { audiovisuales }\end{array}$ & $-0,035$ & 0,436 & 0,305 & $<0,001$ & 0,288 & $<0,001$ & 0,345 & $<0,001$ \\
\hline $\begin{array}{l}\text { Proyectos de } \\
\text { aprendizaje } \\
\text { tutorados }\end{array}$ & $-0,049$ & 0,280 & 0,382 & $<0,001$ & 0,314 & $<0,001$ & 0,360 & $<0,001$ \\
\hline
\end{tabular}

Nota: En negrita las relaciones significativas.

Fuente: Elaboración propia. 
La Tabla 3 recoge los resultados del profesorado sobre la correlación entre el tipo de procesos de calificación aplicados y los instrumentos de evaluación utilizados. En cuanto a la hetero-calificación, aparece una relación significativa y directa con el examen tipo test $(\mathrm{r}=0,139 ; p=0,010)$ e inversa y significativa con el uso del portafolios $(\mathrm{r}=-0,238 ; p<0,001)$, el cuaderno de campo $(\mathrm{r}=-0,148 ; p=0,006)$ y los proyectos de aprendizaje $(\mathrm{r}=-0,255$; $p<0,001)$. En la auto-calificación, existe una relación significativa e inversa con los exámenes tipo test $(\mathrm{r}=-0,143 ; p=0,008)$. En todos los casos, el resultado de la correlación es débil $(\mathrm{r}<0,255)$. No existe relación entre los exámenes de preguntas abiertas, preguntas cortas, preguntas cerradas o dejando disponer de documentos y la auto-calificación (todos, $p>0,05)$. Con el resto de instrumentos, la relación significativa más fuerte se produce con el portafolios, los proyectos de aprendizaje tutorados y los cuadernos de campo. La calificación dialogada no presenta relación significativa ni con los exámenes de preguntas abiertas ni con los de preguntas cerradas $(p>0,05)$. Se relaciona significativa e inversamente con los exámenes tipo test y de preguntas cortas. Con el resto de los ítems se relaciona de forma significativa y positiva con todos, siendo el portafolios y los proyectos de aprendizaje tutorados los que lo hacen con mayor fuerza. En el caso de la calificación entre compañeros, existe una relación positiva y significativa con los exámenes orales, pruebas físicas, portafolios, cuadernos de campo, trabajos escritos, ensayos y proyectos de aprendizaje tutorados $(p<0,002)$, siendo la relación más fuerte con los proyectos tutorados y el portafolios, aunque en todos los casos el valor de la correlación es muy bajo $(\mathrm{r}<0,304)$.

Cuadro 3. Correlación entre instrumentos de evaluación y forma de calificación en profesorado

\begin{tabular}{|c|c|c|c|c|c|c|c|c|}
\hline & \multicolumn{2}{|c|}{$\begin{array}{c}\text { HETERO- } \\
\text { CALIFICACIÓN }\end{array}$} & \multicolumn{2}{|c|}{$\begin{array}{c}\text { AUTO- } \\
\text { CALIFICACIÓN }\end{array}$} & \multicolumn{2}{|c|}{$\begin{array}{c}\text { CAL. } \\
\text { DIALOGADA }\end{array}$} & \multicolumn{2}{|c|}{$\begin{array}{c}\text { CALIFICACIÓN } \\
\text { ENTRE } \\
\text { COMPAÑEROS }\end{array}$} \\
\hline & $\mathbf{r}$ & $p$ & $\mathbf{r}$ & $p$ & $\mathbf{r}$ & $p$ & $\mathbf{r}$ & $p$ \\
\hline Examen tipo test & 0,139 & 0,010 & $-0,143$ & 0,008 & $-0,107$ & 0,047 & 0,007 & 0,891 \\
\hline $\begin{array}{l}\text { Examen de } \\
\text { preguntas abiertas }\end{array}$ & 0,048 & 0,378 & $-0,066$ & 0,222 & $-0,056$ & 0,299 & $-0,059$ & 0,273 \\
\hline $\begin{array}{l}\text { Examen de } \\
\text { preguntas cortas }\end{array}$ & 0,067 & 0,212 & $-0,098$ & 0,070 & $-0,134$ & 0,013 & $-0,073$ & 0,176 \\
\hline $\begin{array}{l}\text { Examen de } \\
\text { preguntas cerradas }\end{array}$ & $-0,007$ & 0,899 & $-0,058$ & 0,285 & $-0,071$ & 0,191 & 0,043 & 0,422 \\
\hline $\begin{array}{l}\text { Exámenes escritos } \\
\text { dejando disponer de } \\
\text { documentos }\end{array}$ & $-0,097$ & 0,071 & 0,090 & 0,095 & 0,131 & 0,015 & 0,052 & 0,335 \\
\hline Exámenes orales & $-0,138$ & 0,011 & 0,154 & 0,004 & 0,215 & $<0,001$ & 0,235 & $<0,001$ \\
\hline $\begin{array}{l}\text { Pruebas prácticas de } \\
\text { carácter físico }\end{array}$ & $-0,093$ & 0,086 & 0,159 & 0,003 & 0,127 & 0,018 & 0,194 & $<0,001$ \\
\hline Portafolios & $-0,238$ & $<0,001$ & 0,302 & $<0,001$ & 0,374 & $<0,001$ & 0,273 & $<0,001$ \\
\hline $\begin{array}{l}\text { Cuadernos de campo } \\
\text { (o fichas de sesiones } \\
\text { prácticas) }\end{array}$ & $-0,148$ & 0,006 & 0,230 & $<0,001$ & 0,252 & $<0,001$ & 0,231 & $<0,001$ \\
\hline $\begin{array}{l}\text { Informes o trabajos } \\
\text { escritos }\end{array}$ & $-0,060$ & 0,268 & 0,132 & 0,015 & 0,116 & 0,032 & 0,165 & 0,002 \\
\hline $\begin{array}{l}\text { Ensayos a partir de } \\
\text { textos o materiales } \\
\text { audiovisuales }\end{array}$ & $-0,144$ & 0,008 & 0,192 & $<0,001$ & 0,204 & $<0,001$ & 0,216 & $<0,001$ \\
\hline $\begin{array}{l}\text { Proyectos de } \\
\text { aprendizaje } \\
\text { tutorados }\end{array}$ & $-0,255$ & $<0,001$ & 0,296 & $<0,001$ & 0,290 & $<0,001$ & 0,304 & $<0,001$ \\
\hline
\end{tabular}

Nota: En negrita las relaciones significativas.

Fuente: Elaboración propia. 


\section{Discusión}

Los resultados de esta investigación muestran que aparecen diferencias significativas en la percepción de profesorado universitario y egresados en todos los instrumentos de evaluación a excepción de los exámenes orales, los exámenes dejando disponer de documentos y los ensayos siendo los egresados los que obtienen valores medios más altos. Estudios como el de Gutiérrez et al. (2013) compararon la opinión de profesores (24), alumnos (43) y egresados (35) de la Licenciatura en Ciencias de la Actividad Física y el Deporte de la Universidad de León sobre la utilización de instrumentos de evaluación. En la comparativa egresados-profesorado, nuestros resultados coinciden con los suyos en lo referente a los diferentes tipos de exámenes, donde al igual que este estudio no encuentran diferencias significativas entre egresados y profesorado en los exámenes dejando disponer de documentos y exámenes orales, y en el resto sí aparecen diferencias significativas siendo los egresados los que presentan valores más altos. Ellos también encuentran diferencias significativas en los ensayos a partir de vídeos o textos, siendo el profesorado el que percibe un mayor uso de este instrumento, mientras que en nuestro estudio no encontramos diferencias significativas en este ítem. Lo que puede deberse a que la muestra recogida por estos autores es pequeña y pertenece a un solo centro universitario. Coincidimos en que todos los grupos piensan que los trabajos escritos se utilizan en bastantes ocasiones.

En el estudio de Hamodi et al. (2015) también realizan una investigación comparativa entre profesorado $(n=14)$, alumnado $(n=205)$ y egresados $(n=46)$ en la Escuela Universitaria de Segovia. Todos los grupos coinciden en que el instrumento más utilizado de promedio son los exámenes escritos de desarrollo, prueba que en nuestro estudio no ha mostrado diferencias significativas entre profesorado y egresados, aunque no es la que valoran de media como la más utilizada. Entre los instrumentos menos utilizados, los egresados, al igual que en nuestro estudio, plantean que la prueba menos utilizada son los exámenes orales, que no coincide con el del profesorado en ninguna de las dos investigaciones.

Por último, en el estudio de Arribas et al. (2016) investigan la utilización de instrumentos de evaluación en una muestra de alumnado $(\mathrm{n}=199)$, profesorado $(\mathrm{n}=53)$ y egresados $(\mathrm{n}=67)$ de Magisterio (Educación Física) y Ciencias de la Actividad Física y el Deporte de las universidades de Valladolid, Salamanca y León. Coincidimos con ellos en que el instrumento más utilizado son los trabajos escritos; sin embargo, no encontramos el mismo consenso que ellos en las pruebas menos utilizadas. Mientras ellos encuentran que para ambos grupos son el examen dejando disponer de materiales, los exámenes orales y las pruebas físicas, nosotros encontramos que los dos primeros son los instrumentos que los egresados menos puntúan de media, y el profesorado puntúa menos de media a las pruebas físicas, pero ambos grupos no coinciden en estos planteamientos.

Si tomamos como referencia investigaciones que hayan focalizado su atención en comparar la visión del profesorado y el alumnado respecto a los instrumentos de evaluación, vemos, al igual que pasa con los egresados, que hay diversidad de opiniones. Martínez et al. (2012), en una muestra de 130 estudiantes y 16 docentes de dos universidades madrileñas de la titulación de Educación Física, encontraron que los instrumentos valorados como los más utilizados eran los exámenes, especialmente los exámenes de preguntas cortas. Estos resultados no coinciden con nuestro estudio donde la prueba más utilizada es el 
trabajo escrito. En el estudio de Ruiz, Ruiz y Ureña (2013), encuentran una coincidencia para ambos grupos entre los instrumentos valorados como los más utilizados, aunque existen diferencias significativas en sus opiniones, siendo los estudiantes los que reportan valores más altos de media en la utilización de los instrumentos.

De forma general, en estos estudios se aprecia que podría haber un uso más variado de los diferentes instrumentos de evaluación, alejados de la concepción tradicional de examen, y estudiados en esta investigación, tal y como se ha reclamado en la aplicación de la evaluación formativa (Dochy et al., 1999). Además, el porcentaje de utilización de los instrumentos de evaluación aún podría ser más alto. En nuestro estudio los participantes reportan que de media todos los instrumentos de evaluación se utilizan bastante o mucho en menos de un $60 \%$ de los casos. Por ello, es necesario seguir aumentando ese porcentaje, así como la variada utilización de instrumentos, de manera que durante la formación inicial del profesorado se puedan valorar todos los tipos de competencias que deben ser desarrolladas por el alumnado universitario. El hecho de que los instrumentos de evaluación más y menos utilizados no coincidan de unos estudios a otros puede deberse a que la mayoría se han recogido en muestras pequeñas en un número reducido de universidades.

En lo referente a los procesos de calificación, resulta llamativa la diferente visión que tiene profesorado y egresados. En nuestro estudio, aunque ambos grupos coinciden en que se utiliza la hetero-calificación más que los procesos de calificación participativos, sí que existen diferencias entre el pensamiento de ambos grupos. Por una parte, los egresados opinan que el profesorado utiliza más la hetero-calificación de lo que ellos piensan, y el profesorado opine que utiliza más las formas de calificación participativas de lo que piensan los egresados. Sin embargo, estos resultados no coinciden con los de otros estudios. Por ejemplo, Gutiérrez et al. (2013), no obtienen diferencias significativas entre la opinión de egresados y profesorado, aunque ambos grupos coinciden, al igual que en nuestro estudio, que se usa bastante más la hetero-calificación que las formas de calificación participativas. Mismos resultados aparecen en el estudio de Hamodi et al. (2015), que también encuentran una baja participación del alumnado en los procesos de calificación, ya sea a través de la auto-calificación, la calificación entre compañeros o la calificación dialogada.

En las investigaciones con profesorado y estudiantes, todas coinciden en la prevalencia de los procesos de hetero-calificación como forma más utilizada para calificar al alumnado durante su formación inicial. (Gutiérrez, Pérez, Pérez y Palacios, 2011; Martínez et al., 2012; Ruiz et al., 2013). Sin embargo, investigaciones como la de Falchikov y Boud (1989) han mostrado que existe una correlación positiva entre la auto-calificación del alumnado y la nota asignada por el profesorado $(\mathrm{r}=0,39)$, resultados que también obtuvieron LópezPastor, Fernández, Santos y Fraile (2012). Estos autores, además, encontraron una alta correlación entre la auto-calificación, la hetero-calificación y la calificación dialogada. La investigación de Falchikov y Goldfinch (2000) ha mostrado idénticos resultados entre la calificación entre compañeros y la nota del profesorado $(\mathrm{r}=0,69)$. Viendo que la participación del alumnado en los procesos de calificación no desvirtúa la nota asignada, parece necesario tomar una mayor conciencia de la importancia que podría tener que el alumnado participe en de la evaluación y la calificación y en la de sus compañeros, ya que las propuestas de calificación en las que participa el alumnado han mostrado tener mayores 
repercusiones para el aprendizaje que cuando la calificación proviene solo del docente (Boud, 1989).

Por otra parte, no hemos encontrado ninguna investigación previa donde se haya relacionado la utilización de una forma de calificación concreta con diferentes instrumentos de evaluación. Nuestra investigación ha mostrado que, los egresados presentan una relación positiva entre la utilización de las formas de calificación participativas y la utilización de la mayoría de los instrumentos de evaluación, mientras que el profesorado relaciona estas formas de calificación inversamente con la utilización de exámenes.

Para nosotros este es un aspecto importante que debe ser investigado, ya que para que haya un cambio significativo en la forma de evaluar en la universidad, no son aspectos aislados los que se deben cambiar, sino que unos cambios deben venir acompañados de otros y estos llevarse a cabo de forma conjunta. En nuestro estudio se ha visto que, aunque se percibe que las formas de calificación participativas se asocian con un mayor número de instrumentos que la hetero-calificación, hay diferencias entre el pensamiento del profesorado y de los egresados. En los procesos de hetero-calificación los egresados solo lo relacionan con los exámenes de preguntas abiertas, mientras que el profesorado lo relaciona de forma directa con los exámenes tipo test e inversamente con el uso de instrumentos como el portafolios, los cuadernos de campo o los proyectos. Las formas de calificación participativas son asociadas por los egresados con el uso de una gran variedad de instrumentos de evaluación, incluyendo desde algunos más tradicionales, como exámenes de preguntas abiertas, preguntas cortas, preguntas cerradas, etc., a otros con un perfil más innovador, como son los proyectos o el portafolios. El profesorado, por su parte, relaciona algunas de estas formas de calificación de forma inversa con exámenes tipo test (auto-calificación y calificación dialogada), exámenes de preguntas cortas (calificación dialogada), dando a entender que, para ellos, la utilización de estas formas de calificación es incompatible con la utilización de instrumentos de evaluación de un tinte más tradicional como son los exámenes.

Las investigaciones muestran que se va produciendo un avance en la aplicación de procesos de evaluación formativa en las aulas universitarias, sin embargo, aún predomina un enfoque tradicional (López-Pastor, Manrique y Vallés, 2011; Panadero, Fraile, Fernández, Castilla-Esteve y Ruiz, 2019), cuando parece necesaria una mayor aplicación de la evaluación con más y mejores instrumentos que permitan demostrar los aprendizajes. Se sigue igualando la evaluación a la calificación con prácticas que buscan principalmente un objetivo de certificación y rendición de cuentas (Valverde y Ciudad, 2014), y en las que el alumnado no es un protagonista activo de las prácticas de evaluación y calificación.

Esta investigación presenta ciertas fortalezas, como el amplio número de participantes, el planteamiento de un objetivo de investigación novedoso, así como la utilización de un instrumento que ha sido previamente validado por un grupo de expertos en el ámbito de la evaluación formativa. Sin embargo, también presenta algunas limitaciones en lo referente, principalmente, al tipo de muestreo empleado, ya que al ser incidental no permite generalizar los resultados. El diseño transversal de esta investigación también representa una limitación. Además, también se valora la percepción del profesorado y egresados del empleo de los instrumentos de evaluación y las formas de calificación, y no el verdadero grado de empleo de estas. 


\section{Conclusiones}

Esta investigación pone de manifiesto la diferente percepción existente entre docentes universitarios y egresados en lo que a utilización de instrumentos de evaluación y formas de calificación empleadas se refiere, destacando la mejor valoración de los egresados en lo que a uso de los diferentes instrumentos de evaluación se refiere y el hecho de que el profesorado considera que utiliza formas de calificación más participativas de lo que perciben los egresados. Además, a través de este estudio hemos podido valorar que, tanto docentes universitarios como egresados, perciben una relación entre la utilización de formas de calificación participativas (auto-calificación, calificación entre compañeros y calificación compartida) con la utilización de una gran variedad de instrumentos de evaluación, sugiriendo que, durante la formación inicial, el profesorado que decide emplear procedimientos de evaluación formativa busca hacerlo a través de todos los elementos que forman parte del proceso. Mientras que en la utilización de procesos de calificación tradicionales como es la hetero-calificación no aparece una relación clara con ningún instrumento de evaluación. Consideramos que es necesario realizar más investigaciones que profundicen en la forma en que los procesos de evaluación formativa se emplean en el aula universitaria, analizando no solo que tipo de instrumentos o formas de calificación se emplean, sino cuáles son las causas para emplear unos y no otros.

\section{Agradecimientos}

Proyecto "Las competencias docentes en la formación inicial del profesorado de Educación Física” financiado por el plan estatal de Investigación Científica y Técnica y de Innovación 2013-2016 del Plan I+D+I (REF EDU2013-42024-R).

\section{Referencias}

Álvarez, J.M. (2003). La evaluación a examen. Ensayos críticos. Buenos Aires: Miño y Dávila.

American Psychological Association. (2010). Publication manual of the American Psychological Association. Washington, DC: Autor.

Arribas, J.M., Manrique, J.C. y Tabernero, B. (2016). Instrumentos de evaluación utilizados en la formación inicial del profesorado y su coherencia para el desarrollo de competencias profesionales en los estudiantes: visión del alumnado, egresados y profesorado. Revista Complutense de Educación, 27(1), 237-255.

Biggs, J., y Tang, C. (2007). Teaching for Quality Learning at University. What the Student Does. Berkshire: McGraw-Hill, SRHE and Open University Press.

Black, P. y Wiliam, D. (1998). Assessment and classroom learning. Assessment in Education: Principles, Policy y Practice, 5(1), 7-74. https://doi.org/10.1080/0969595980050102

Black, P. y Wiliam, D. (2009). Developing the theory of formative assessment. Educational Assessment, Evaluation and Accountability, 21, 5-31. https://doi.org/10.1007/s1 1092-008-9068-5

Bonsón, M. y Benito, A. (2007). Evaluación y aprendizaje. En Benito, A. y Cruz, A. (Coords). Nuevas claves para la docencia universitaria en el Espacio Europeo de Educación Superior. Madrid: Narcea. 
Boud, D. (1989). The role of self-assessment in student grading. Assessment y Evaluation in Higher Education, 14(1), 20-30. https://doi.org/10.1080/0260293890140103

Brown, S. (2004). Assessment for Learning. Learning and Teaching in Higher Education, 1, 81-89.

Brown, S. y Glasner, A. (Eds.). (2007). Evaluar en la Universidad. Problemas y nuevos enfoques. Madrid: Narcea.

Calderón, C. y Escalera, G. (2008). La evaluación de la docencia ante el reto del Espacio Europeo de Educación Superior (EEES). Educación XX1, 11, 237-256. https://doi.org/10.5944/educxx 1.11.0.316

Campbell, C. y Van der Wende, M. (2000). International Initiatives and Trends in Quality Assurance for European Higher Education. Helsinki: The European Network for Quality Assurance in Higher Education.

Cañadas, L., Santos-Pastor, M. L y Castejón, F. J. (2018a). Desarrollo de Competencias Docentes en la Formación Inicial del Profesorado de Educación Física. Relación con los Instrumentos de Evaluación. Estudios Pedagógicos, 44(2), 111-126.

Cañadas, L., Santos-Pastor, M. L. y Castejón, F.J. (2018b). Evaluación en la formación inicial: ¿Avance o retroceso? Bordón. Revista de Pedagogía, 70(4), 9-22. https://doi.org/10.13042/Bordon.2018.64434

Castejón, F. J., Santos-Pastor, M. L. y Palacios, A. (2015). Escala sobre metodologías participativas y evaluación formativa en la formación inicial en educación física. Revista Internacional de Medicina y Ciencias de la Actividad Física y el Deporte, 15(58), 245-267. https://doi.org/10.15366/rimcafd2015.58.004

Castejón, F.J., Capllonch, M., González, N. y López-Pastor, V. M. (2009). Técnicas e instrumentos de evaluación. En V. M. López-Pastor (Ed.), La Evaluación Formativa y Compartida en Educación Superior: propuestas, técnicas, instrumentos y experiencias (pp. 65-92). Madrid: Narcea.

Comisión Europea. (2004). Sistema europeo de transferencia y acumulación de créditos (ECTS). Luxemburgo: Oficina de Publicaciones Oficiales de las Comunidades Europeas.

Comisión Europea (2011). Guía para el uso de ECTS. Luzemburgo: Organismo Autónomo Programas Educativos Europeos.

Crooks, T. J. (1988). The impact of classroom evaluation practices on students. Review of Educational Research, 58(4), 438-481 https://doi.org/10.3102/00346543058004438

Delgado, A. M. (2005). Competencias y diseño de la evaluación continua y final en el Espacio Europeo de Educación Superior. Madrid: Dirección General de Universidades.

Dochy, F., Segers, M. y Sluijsman, D. (1999). The Use of Self-, Peer and Co-assessment in Higher Education: a review. Studies in Higher Education, 24(3), 331-350. https://doi.org/10.1080/03075079912331379935

Espinosa, J. K., Jiménez, J., Olabe, M. y Basogain, X. (julio, 2006). Innovación docente para el desarrollo de competencias en el EEES. VIII Congreso de Tecnologías Aplicadas a la Enseñanza de la Electrónica. Madrid.

European Communities. (2009). ECTS Users' Guide. Bruselas: European Communities.

Falchikov, N. y Boud, D. (1989). Student Self-Assessment in Higher Education: A Meta-Analysis. Review of Educational Research, 59(4), 395-430. https://doi.org/10.3102/00346543059004395 
Falchikov, N. y Goldfinch, J. (2000). Student Peer Assessment in Higher Education: A MetaAnalysis Comparing Peer and Teacher Marks. Review of Educational Research, 70(3), 287322. https://doi.org/10.3102/00346543070003287

Gessa, A. (2011). La coevaluación como metodología complementaria de la evaluación del aprendizaje. Análisis y reflexión en las aulas universitarias. Revista de Educación, 354, 749764

González, I. (2006). Dimensiones de evaluación de la calidad universitaria en el Espacio Europeo de Educación Superior. Revista Electrónica de Investigación Psicoeducativa, 4(3), 445-468

Gutiérrez, C., Pérez, A. y Pérez, M. (2013). Percepciones de profesores, alumnos y egresados sobre los sistemas de evaluación en estudios universitarios de formación del profesorado de Educación Física. Ágora para la Educación Física y el Deporte, 15(2), 130-151.

Gutiérrez, C., Pérez, A., Pérez, M. y Palacios, A. (2011). Percepciones de profesores y alumnos sobre la enseñanza, evaluación y desarrollo de competencias en estudios universitarios de formación de profesorado. Cultura y Educación, 23(4), 499-514.

https://doi.org/10.1174/113564011798392451

Hamodi, C., López-Pastor, V. M, y López, A. T. (2015). Medios, técnicas e instrumentos de evaluación formativa y compartida del aprendizaje en educación superior. Perfiles Educativos, 37(147), 147-161. https://doi.org/10.1016/j.pe.2015.10.004

Heritage, M. (2007). Formative Assessment: What Do Teachers Need to Know and Do? Phi Delta Kappan, 89(2), 140-145. https://doi.org/10.1177/003172170708900210

Ibarra, M. S. y Rodríguez, G. (2014). Modalidades participativas de evaluación: Un análisis de la percepción del profesorado y de los estudiantes universitarios. Revista de Investigación Educativa, 32(2), 339-361. https://doi.org/018/rie.32.2.172941

Ibarra, M. S., Rodríguez, G. y Gómez, M. A. (2012). La evaluación entre iguales: beneficios y estrategias para su práctica en la universidad. Revista de Educación, 359, 206-231. https://doi.org/10.4438/1988-592X-RE-2011-359-092

López-Pastor, V. M. (2006). El papel de la evaluación formativa en el proceso de convergencia hacia el E.E.E.S. Análisis del estado de la cuestión y presentación de un sistema de intervención. Revista Interuniversitaria de Formación del Profesorado, 20(3), 93-119.

López-Pastor, V. M. (2017). Evaluación formativa y compartida: evaluar para aprender y la implicación del alumnado en los procesos de evaluación y aprendizaje. En V. M. LópezPastor y Á. Pérez-Pueyo (Coords). Evaluación formativa y compartida en educación: experiencias de éxito en todas las etapas educativas (pp. 34-68). León: Universidad de León.

López-Pastor, V. M., Manrique, J. C. y Vallés, C. (2011). La evaluación y la calificación en los nuevos estudios de Grado. Especial incidencia en la formación inicial del profesorado. Revista Electrónica Interuniversitaria de Formación del Profesorado, 14(4).

López-Pastor, V. M., Martínez, L. F. y Julián, J. A. (2007). La Red de Evaluación Formativa, Docencia Universitaria y Espacio Europeo de Educación Superior (EEES). Presentación del proyecto, grado de desarrollo y primeros resultados. Revista de Docencia Universitaria RED$U, 2$.

López-Pastor, V. M., Fernández, J. M., Santos, M. L. y Fraile, A. (2012). Students' self-grading, professor's grading and negotiated final grading at three university programmes: analysis of reliability and grade difference ranges and tendencies. Assessment y Evaluation in Higher Education, 37(4), 353-364. https://doi.org/10.1080/02602938.2010.545868 
López-Pastor, V. M., Castejón, J., Sicilia, A., Navarro, V. y Webb, G. (2011). The process of creating a cross-university network for formative and shared assessment in higher education in Spain and its potential applications. Innovations in Education and Teaching International, 48(1), 79-90. https://doi.org/10.1080/14703297.2010.543768

Martínez, L. F., Castejón, F. J. y Santos-Pastor, M. L. (2012). Diferentes percepciones sobre evaluación formativa entre profesorado y alumnado en formación inicial en educación física. Revista Electrónica Interuniversitaria de Formación del Profesorado, 15(4).

Padilla, M. T. y Gil, J. (2008). La evaluación orientada al aprendizaje en la Educación Superior: condiciones y estrategias para su aplicación en la docencia universitaria. Revista Española de Pedagogía, 241, 467-486.

Palomares, A. (2007). Nuevos retos educativos. El modelo docente en el espacio europeo. Cuenca: Ediciones de la Universidad de Castilla-la-Mancha.

Panadero, E., Fraile, J., Fernández, J., Castilla-Estévez, D. y Ruíz, M. A. (2019). Spanish university assessment practices: examination tradition with diversity by faculty. Assessment छ Evaluation in Higher Education, 44(3), 379-397. https://doi.org/10.1080/02602938.2018.1512553

Real Decreto 1393/2007, de 29 de octubre, por el que se establece la ordenación de las enseñanzas universitarias oficiales.

Red de Evaluación Formativa y Compartida en Educación Superior. https://redevaluacionformativa.wordpress.com/

Rodríguez, G., Ibarra, E. y García, M. S. (2013). Autoevaluación, evaluación entre iguales y coevaluación: conceptualización y práctica en las universidades españolas. Revista de Investigación en Educación, 1 1(2), 198-210.

Ruiz, J. R., Ruiz, E. y Ureña, N. (2013). La evaluación en la formación inicial del profesorado: qué creemos hacer y qué perciben los alumnos. Cultura, 8, 17-29.

Sadler, R. (1989). Formative assessment and the design of instructional systems. Instructional Science, 18, 119-144. https://doi.org/10.1007/BFo0117714

Sambell, K., McDowell, L. y Montgomery, C. (2013). Assessment for learning in Higher Education. Londres: Routledge

Stiggins, R. (2005). Assessment for Learning: A Path to Success in Standards-Based Schools. Phi Delta Kappan, 87(4), 324-328. https://doi.org/10.1177/003172170508700414

Trillo, F. y Porto, N. (1999). La percepción de los estudiantes sobre evaluación en la Universidad. Un estudio de caso en la Facultad de Ciencias de la Educación. Revista de Innovación Educativa, 9, 55-75.

Valverde, J. y Ciudad, A. (2014). El uso de e-rúbricas para la evaluación de competencias en estudiantes universitarios. Estudio sobre fiabilidad del instrumento. Revista de Docencia Universitaria, 12(1), 49-79.

Wiliam, D. (2006). Formative Assessment: Getting the Focus Right. Educational Assessment, 11(34), 283-289. https://doi.org/10.1080/10627197.2006.9652993

Yorke, M. (2003). Formative assessment in higher education: Moves towards theory and the enhancement of pedagogic practice. Higher Education, 45, 477-501.

https://doi.org/10.1023/A:1023967026413 


\section{Cv de los autores}

\section{Laura Cañadas Martín}

Doctora con Mención Internacional en Ciencias de la Actividad Física y el Deporte por la Universidad Autónoma de Madrid con un contrato predoctoral de formación docente e investigadora. Su trayectoria investigadora se centra en las competencias docentes y la evaluación formativa en Educación Superior. ORCID ID. 0000-0003-4179-9018. Email: laura.cannadas@uam.es

\section{María Luisa Santos-Pastor}

Profesora titular del Departamento de Educación Física, Deporte y Motricidad Humana en la Universidad Autónoma de Madrid. Sus principales publicaciones y líneas de investigación se centran en la docencia, evaluación e innovación en educación superior. ORCID ID: 0000-0002-4985-0810. Email:marisa.santos@uam.es

\section{Francisco Javier Castejón Oliva}

Profesor titular del Departamento de Educación Física, Deporte y Motricidad Humana en la Universidad Autónoma de Madrid. Sus principales líneas y trabajos de investigación se centran en la docencia, evaluación e innovación en educación superior. ORCID ID: 0000o001-7507-6091. Email: javier.castejon@uam.es 